\title{
Relationship between oral clinical conditions and daily performances
}

\section{Andréa Silveira Gomes ${ }^{(a)}$ \\ Claides Abegg ${ }^{(b)}$ \\ Jandyra Maria Guimarães Fachel(c)}

(a) MSc student in Collective Oral Health; (b)PhD, Professor - Department of Social and Preventive Dental Sciences, School of Dentistry, Federal University of Rio Grande do Sul, Porto Alegre, RS, Brazil.

(c) PhD, Professor, Department of Statistics, Mathematics Institute - Federal University of Rio Grande do Sul, Porto Alegre, RS, Brazil.

Corresponding author:

Andréa Silveira Gomes

Av. Ipiranga, 3491, Apto. 711, Santana

Porto Alegre - RS - Brazil

CEP: 90610-001

E-mail:andreag@terra.com.br

Received for publication on Feb 14, 2007

Accepted for publication on Oct 24, 2007

\begin{abstract}
The aim of this study was to assess the impact of oral status on the daily performances of civil servants from the Public Works and Waste Management Department of the city of Porto Alegre, located in Southern Brazil. A cross-sectional study was conducted on a representative sample composed of 276 civil servants with ages ranging from 35 to 44 years. The Oral Impacts on Daily Performances index developed was employed to measure impacts caused by oral clinical conditions. Oral examinations were performed after the interviews. Multinomial Logistic Regression Analysis was used. After adjusting for sex and educational level, the results showed that the subjects with high DMFT scores were 5.8 times $(95 \% \mathrm{CI}=2.1-16.1)$ more likely to have high impacts on their everyday life than those with low DMFT scores. Subjects that presented some coronal caries were 4.3 times $(95 \% \mathrm{CI}=1.9-9.8)$ more likely to have high impacts on their everyday life than those with no coronal caries. Dental status assessed through the DMFT index and coronal caries are important indicators of impacts on the everyday life of the studied population.
\end{abstract}

Descriptors: Oral health; Quality of life; Indicators; DMF Index. 


\section{Introduction}

Nowadays, oral health is understood as a dentition which is comfortable, functional, and with such an appearance that allows people to perform their social function and daily activities without physical, psychological or social inconveniences. ${ }^{1}$

The use of socio-dental indicators based on selfperception and clinical impacts offers important advantages for planning and providing dental services, thus changing the emphasis from merely biological to social and psychological aspects. ${ }^{2}$

Different socio-dental indicators were developed over the past few years and one of them is the Oral Impacts on Daily Performances (OIDP). ${ }^{3}$

The OIDP is a socio-dental indicator based conceptually on the International Classification of Impairments, Disabilities and Handicaps created by the $\mathrm{WHO}^{4}$ in 1980. The OIDP consists of eight daily performances: eating and enjoying food; speaking and pronouncing clearly; cleaning your teeth; sleeping and relaxing; smiling, laughing, and showing your teeth without embarrassment; maintaining your emotional state without becoming upset; carrying out your major work or social role; and enjoying contact with other people. ${ }^{3}$ One of its goals is to assess the frequency and severity of the impacts that affect individual daily activities. In order to give more representativeness to the OIDP in the evaluation of specific treatment needs, the individual is asked about the major symptom perceived and the main oral condition responsible for the impact in his/her daily activities, attempting to establish a relation between impact and clinical condition. ${ }^{3}$

According to Sheiham $^{2}$ (2000), associating sociodental indicators that assess oral health self-perception and impacts caused by oral conditions on the quality of life with clinical evaluation of oral health conditions may offer a more comprehensive view of oral health status and treatment needs.

This study aims to assess the relationship between socio-demographic factors, clinical conditions and the impacts caused by oral clinical conditions on the daily performances of civil servants with ages ranging from 35 to 44 years from the Public Works and Waste Management Department of the city of Porto Alegre, Brazil.

\section{Material and Methods}

This cross-sectional study was carried out using a sample of the 728 civil servants from the Public Works and Waste Management Department of Porto Alegre with ages ranging from 35 to 44 years. The size of the sample was calculated based on an expected prevalence of $50 \%$ and $95 \%$ confidence level. A minimum sample size of 251 people was defined and further increased in $10 \%$ to compensate possible losses. The target-population was divided considering the workers' occupation in the administrative and operational areas to insure different education and income levels in the study sample. A total of two hundred and seventy-six civil servants participated in the interview and clinical exam. Both exams were performed on the same day.

The questionnaire included socio-demographic variables and the Oral Impacts on Daily Performances (OIDP) index, already used by Cortes et al. ${ }^{5}$ (2002) and Michel-Crosato et al. ${ }^{6}$ (2005) over a Brazilian population sample.

Each individual was asked the following question: During the past six months, was there any problem about your oral health that caused you difficulties with eating and enjoying food, speaking and pronouncing clearly, cleaning your teeth, sleeping and relaxing, smiling, laughing, and showing your teeth without embarrassment, maintaining your emotional state without becoming upset, carrying out your major work or social role, and/or enjoying contact with other people? The possible answers were yes and no. The affected daily performances were then classified according to how often they had had this difficulty (impact frequency):

1. Less than once a month or intervals of up to 5 days;

2. about 1 to 2 times a month or intervals of up to 15 days;

3. about 1 to 2 times a week or intervals of up to 30 days;

4. 3 to 4 times a week or intervals of up to 3 months;

5. every day or nearly every day, or intervals of more than 3 months.

The affected daily performances were also classified according to how much effect this difficulty had 
had on his/her everyday life (degree of severity):

1. No effect;

2. a very minor effect;

3. a fairly minor effect;

4. a moderate effect;

5. a fairly severe effect;

6. a very severe effect.

Every worker who noticed any impact on his/her performance was asked about whether the major symptom was pain, discomfort, work limitation, dissatisfaction with own appearance or other. They were then asked to specify the main oral condition that, from their point of view, had caused this difficulty on their daily performance.

The OIDP scores were formed multiplying the impact frequency by the degree of severity in each affected performance and then the performance scores were added, thus establishing the individual OIDP.

A trained examiner (dental practitioner) performed the clinical examination according to the $\mathrm{WHO}^{7}$ criteria using the DMFT index under artificial illumination (flashlight). The values of the intra-examiner Kappa in the calibration exercise were 0.91 for coronal caries. A pilot study was carried out in order to assess the techniques developed for the main study.

After finishing the data collection, every tenth subject had a duplicate examination. A total of 20 subjects were re-examined and the values of intra-examiner Kappa in the re-exams were 0.92 for coronal caries. Both the Research Ethics Committee from the Federal University of Rio Grande do Sul, Brazil, and the Board of Directors from the Public Works and Waste Management Department of Porto Alegre gave their approval to the data collection.

The variable OIDP, the outcome category, was initially divided into two categories $(O I D P=0 /$ OIDP $>0$ ). The variable OIDP $>0$ was divided into tertiles according to frequency distribution resulting in Low OIDP, Medium OIDP and High OIDP. The variable coronal caries was dichotomized in none and 1 or more, and DMFT was divided into tertiles according to frequency distribution (Low DMFT, Medium DMFT and High DMFT). The socio-demographic variables used were sex (female/male), education (measured in terms of years of completed schooling without repetition and dichotomized according to the mean, corresponding to 6 years of schooling) and monthly income (measured in terms of minimum-wage units and dichotomized using the median as a cut-off point).

In order to evaluate the effect of independent variables on OIDP, Multinomial Logistic Regression Analysis was used, estimating values for odds ratio (OR) and the respective 95\% confidence interval. The category OIDP = 0 was chosen as the outcome reference category. A chi-square test was performed prior to the regression analysis.

At a first stage a simple multinomial logistic regression was made with the variables that presented $\mathrm{p} \leq 0.25$ in the chi-square test.

At a second stage a multiple multinomial logistic regression was performed using all socio-demographic and clinical variables simultaneously according to the $\mathrm{p} \leq 0.25$ criteria recommended by Hosmer, Lemeshow ${ }^{8}$ (2000). The OR and 95\% CI estimates for each variable were adjusted for the effect of all the other variables studied in the model. A manual stepwise procedure with backward exclusion was used.

The variable income, though showing no significant association, was firstly included in the model. It was further eliminated from the final model since no other independent variables were affected.

The model included the explanatory variables sex, education, coronal caries and DMFT. The outcome reference category was $O I D P=0$.

\section{Results}

There was a high prevalence of impacts caused by oral conditions on the daily performances of adults (Table 1).

There was no significant association between clinical and socio-demographic variables for Low $O I D P$ in relation to $O I D P=0$. The presence of one or more coronal caries was the only variable significantly associated to Medium OIDP. As for High $O I D P$, all variables presented, after adjusting, an increase in the likelihood of high impact on daily performances (Table 2). 
Table 1 - Frequencies of socio-demographic variables, clinical variables and OIDP ( $\mathrm{n}=276)$. Porto Alegre, RS, 2005.

\begin{tabular}{c|c|c}
\hline \multirow{2}{*}{ Sex } & Variables & $\mathrm{n}(\%)$ \\
\cline { 2 - 3 } & Male & $201(72.82)$ \\
\hline \multirow{3}{*}{ Education } & Female & $75(27.17)$ \\
\cline { 2 - 3 } & Years of schooling $\leq 6$ years & $140(50.72)$ \\
\hline \multirow{3}{*}{ Income } & Income $\leq 4 \mathrm{MW}$ & $141(51.08)$ \\
\cline { 2 - 3 } & Income $>4 \mathrm{MW}$ & $135(48.91)$ \\
\hline \multirow{2}{*}{$\begin{array}{c}\text { Coronal } \\
\text { caries }\end{array}$} & None & $142(51.55)$ \\
\hline \multirow{3}{*}{ DMFT } & 1 or more & $134(48.44)$ \\
\cline { 2 - 3 } & Low (0-12) & $83(30.07)$ \\
\cline { 2 - 3 } & Medium (13-19) & $98(35.50)$ \\
\hline \multirow{3}{*}{ OIDP } & High (20-32) & $95(34.45)$ \\
\cline { 2 - 3 } & Low (1-15) & $65(23.55)$ \\
\cline { 2 - 3 } & Medium (16-45) & $67(24.27)$ \\
\cline { 2 - 3 } & Total OIDP (46-200) $>0$ & $65(23.55)$ \\
\hline
\end{tabular}

$M W:$ minimum wages.

\section{Discussion}

The present study showed that the oral clinical status is related to physical, psychological and social impacts on the daily performances of adults. Some variations on high, medium and low impact levels were observed according to the levels of oral health measured by DMFT. Clinical variables, High DMFT $(\mathrm{OR}=5.8)$ and presence of one or more coronal caries $(\mathrm{OR}=4.3)$ were found to be strong indicators of high impact on daily performances.

However, considering the high prevalence of the outcome, as well as the design of the study, the odds ratio should be considered with caution, even though they might be considered valid indicators of a higher chance of impact on the daily performances of the exposed subjects.

These results are supported by other studies which confirm the presence of oral disease as an oral impact indicator, indicating that the worse the oral clinical condition, the higher the social impact. ${ }^{9-12}$ Studies about social impact related to DMFT ${ }^{13}$ and untreated caries ${ }^{12}$ showed a significant relation between poor clinical conditions and high impact on daily performances levels as well.
Some factors play a role on the perception of impact caused by oral clinical conditions. The DMFT, the most widely used index in oral health condition clinical evaluation, both in academic works and in treatment need evaluations, ${ }^{2}$ considers its components equally relevant. The individuals, however, perceive these components as having different impacts on their daily performances. ${ }^{13}$

Moreover, a number of oral diseases, being asymptomatic, are frequently unknown to the individuals and thus interfere in their perception of impact caused by oral clinical conditions. ${ }^{13}$ Individuals refer more to acute problems rather than chronic ones because acute problems are more prone to cause pain and discomfort, ${ }^{3}$ interfering more in their daily activities $^{13}$ than chronic oral health problems.

Cultural factors related to social norms about the concept of oral health play a role on the perception of impact caused by oral clinical conditions ${ }^{12}$ as well, and may have a greater influence than social class on the determination of oral conditions. ${ }^{14}$

An association between levels of impact and sex and education had been expected to be found. This result is consistent with that of other studies showing that women suffer and perceive more impacts caused by oral clinical conditions than men. ${ }^{14,15}$

An explanation to such differences between women and men may be related to the perception and values of oral health in addition to specific conditions like hormonal changes and greater use of medicaments, ${ }^{15}$ which can influence women's oral health.

Some studies show that poorly educated individuals present a higher incidence of impacts caused by oral clinical conditions, ${ }^{16}$ and social conditions ${ }^{12-}$ ${ }^{14}$ present a significant association with a higher incidence and perception of impacts caused by oral clinical conditions.

A possible explanation for this association is that poorly educated individuals from lower income families have worse living and working conditions. These factors may affect their habits and access to educational and preventive methods regarding health and dental treatment.

In this study, no statistically significant association between impact caused by oral clinical condi- 
Table 2 - Odds ratios $(\mathrm{OR})$ and $95 \%$ confidence intervals $(95 \% \mathrm{Cl})$ between socio-demographic and clinical variables and OIDP. Multinomial Logistic Regression Model with OIDP as dependent variable and OIDP $=0$ as reference category $(\mathrm{n}=276)$. Porto Alegre, RS, 2005.

\begin{tabular}{|c|c|c|c|c|c|c|c|}
\hline & \multirow[b]{2}{*}{ Variables } & \multicolumn{2}{|c|}{ Low OIDP } & \multicolumn{2}{|c|}{ Medium OIDP } & \multicolumn{2}{|c|}{ High OIDP } \\
\hline & & $\begin{array}{c}\text { OR } \\
(95 \% \mathrm{Cl})\end{array}$ & $\begin{array}{c}\text { Adjusted OR } \\
(95 \% \mathrm{Cl})\end{array}$ & $\begin{array}{c}\text { OR } \\
(95 \% \mathrm{Cl})\end{array}$ & $\begin{array}{c}\text { Adjusted OR } \\
(95 \% \mathrm{Cl})\end{array}$ & $\begin{array}{c}\text { OR } \\
(95 \% \mathrm{Cl})\end{array}$ & $\begin{array}{l}\text { Adjusted OR } \\
(95 \% \mathrm{Cl})\end{array}$ \\
\hline \multirow[b]{2}{*}{ Sex } & Male & 1 & 1 & 1 & 1 & 1 & 1 \\
\hline & Female & $\begin{array}{c}0.984 \\
(0.4-2.2)\end{array}$ & $\begin{array}{c}1.148 \\
(0.4-2.7)\end{array}$ & $\begin{array}{c}1.235 \\
(0.5-2.7)\end{array}$ & $\begin{array}{c}1.582 \\
(0.6-3.7)\end{array}$ & $\begin{array}{c}3.375 \\
(1.6-7.0)^{* *}\end{array}$ & $\begin{array}{c}\mathbf{6 . 6 1 7} \\
(2.6-16.3)^{*}\end{array}$ \\
\hline \multirow[b]{2}{*}{ Education } & Years of schooling $>6$ & 1 & 1 & 1 & 1 & 1 & 1 \\
\hline & Years of schooling $\leq 6$ & $\begin{array}{c}0.962 \\
(0.4-1.8)\end{array}$ & $\begin{array}{c}1.228 \\
(0.5-2.6)\end{array}$ & $\begin{array}{c}1.029 \\
(0.5-1.9)\end{array}$ & $\begin{array}{c}1.065 \\
(0.4-2.2)\end{array}$ & $\begin{array}{c}\mathbf{2 . 5 0 3} \\
(1.2-4.9)^{* * *}\end{array}$ & $\begin{array}{c}\mathbf{6 . 5 7 4} \\
(2.4-17.6)^{*}\end{array}$ \\
\hline \multirow{2}{*}{$\begin{array}{l}\text { Coronal } \\
\text { caries }\end{array}$} & None & 1 & 1 & 1 & 1 & 1 & 1 \\
\hline & 1 or more & $\begin{array}{c}1.543 \\
(0.7-3.0)\end{array}$ & $\begin{array}{c}1.525 \\
(0.7-3.1)\end{array}$ & $\begin{array}{c}\mathbf{2 . 6 7 1} \\
(1.3-5.2)^{* * *}\end{array}$ & $\begin{array}{c}\mathbf{2 . 8 3 4} \\
(1.3-5.8)^{* * * *}\end{array}$ & $\begin{array}{c}3.722 \\
(1.8-7.4)^{*}\end{array}$ & $\begin{array}{c}4.374 \\
(1.9-9.8)^{*}\end{array}$ \\
\hline \multirow{3}{*}{ DMFT } & Low & 1 & 1 & 1 & 1 & 1 & 1 \\
\hline & Medium & $\begin{array}{c}1.027 \\
(0.4-2.2)\end{array}$ & $\begin{array}{c}0.949 \\
(0.4-2.1)\end{array}$ & $\begin{array}{c}1.446 \\
(0.6-3.1)\end{array}$ & $\begin{array}{c}1.138 \\
(0.5-2.5)\end{array}$ & $\begin{array}{c}2.381 \\
(0.9-6.0)\end{array}$ & $\begin{array}{c}1.555 \\
(0.5-4.2)\end{array}$ \\
\hline & High & $\begin{array}{c}1.071 \\
(0.4-2.4)\end{array}$ & $\begin{array}{c}1.094 \\
(0.4-2.6)\end{array}$ & $\begin{array}{c}1.429 \\
(0.6-3.2)\end{array}$ & $\begin{array}{c}1.285 \\
(0.5-3.1)\end{array}$ & $\begin{array}{c}\mathbf{5 . 7 1 4} \\
(2.2-14.3)^{*}\end{array}$ & $\begin{array}{c}\mathbf{5 . 8 3 8} \\
(2.1-16.1)^{* *}\end{array}$ \\
\hline
\end{tabular}

${ }^{*} p<0.001 ;{ }^{* *} p=0.001 ;{ }^{* * *} p<0.05 ;{ }^{* * * *} p=0.05$

tions and income was observed, probably due to the fact that there were no large income differences between workers from the administrative and the operational area.

\section{Conclusions}

This study showed that oral problems assessed by the DMFT index as well as "coronal caries" are indicators of impact on oral health in the adult population along with sex and education. The finding that the higher the DMFT index, the higher the level of impact on daily performances indicates that, for adults, DMFT levels are directly related to levels of physical, psychological and social impacts on daily performances. Yet, the individual components of

\section{References}

1. Yewe-Dyer M. The definition of oral health. Br Dent J. 1993;174(7):224-5.

2. Sheiham A. A determinação de necessidades de tratamento odontológico: uma abordagem social. In: Pinto VG, editor. Saúde Bucal Coletiva. São Paulo: Santos; 2000. p. 223-50. the DMFT index must be taken into consideration since every component is considered to be equally relevant in spite of causing different impacts on daily performances.

In this study, statistical tests showed that poorly educated female subjects with high DMFT scores and untreated caries are more susceptible to physical, psychological and social impacts on their daily performances than males with better oral clinical conditions and higher education.

This new approach of oral health assessment can be used by clinicians, in academic works, epidemiological surveys and oral health evaluation programs, showing to a full extent the influence of dental problems on individuals' lives. 
5. Cortes MI, Marcenes W, Sheiham A. Impact of traumatic injuries to the permanent teeth on the oral health-related quality of life in 12-14-year-old children. Community Dent Oral Epidemiol. 2002;30(3):193-8.

6. Michel-Crosato E, Biazevic MG, Crosato E. Relationship between dental fluorosis and quality of life: a population based study. Braz Oral Res. 2005;19(2):150-5.

7. OMS - Organização Mundial da Saúde. Levantamentos Básicos em Saúde Bucal. $4^{a}$ ed. São Paulo: Santos; 1999.

8. Hosmer DW, Lemeshow S. Applied logistic regression. $2^{\text {nd }}$ ed. New York: John Wiley \& Sons; 2000.

9. Biazevic MG, Michel-Crosato E, Iagher F, Pooter CE, Correa SL, Grasel CE. Impact of oral health on quality of life among the elderly population of Joaçaba, Santa Catarina, Brazil. Braz Oral Res. 2004;18(1):85-91.

10. Pires CP, Ferraz MB, de Abreu MH. Translation into Brazilian Portuguese, cultural adaptation and validation of the oral health impact profile (OHIP-49). Braz Oral Res. 2006;20(3):263-8.
11. Robinson PG, Gibson B, Khan FA, Birnbaum W. Validity of two oral health-related quality of life measures. Community Dent Oral Epidemiol. 2003;31(2):90-9.

12. Slade GD, Spencer AJ, Locker D, Hunt RJ, Strauss RP, Beck JD. Variations in the social impact of oral conditions among older adults in South Australia, Ontario, and North Carolina. J Dent Res. 1996;75(7):1439-50.

13. Leao A, Sheiham A. Relation between clinical dental status and subjective impacts on daily living. J Dent Res. 1995;74(7):1408-13.

14. Sheiham A, Steele JG, Marcenes W, Tsakos G, Finch S, Walls AW. Prevalence of impacts of dental and oral disorders and their effects on eating among older people; a national survey in Great Britain. Community Dent Oral Epidemiol. 2001;29(3):195-203.

15. Mc Grath C, Bedi R. Gender variations in the social impact of oral health. J Ir Dent Assoc. 2000;46(3):87-91.

16. Srisilapanan P, Sheiham A. The prevalence of dental impacts on daily performances in older people in Northern Thailand. Gerodontology. 2001;18(2):102-8. 